\title{
MYC Association with Cancer Risk and a New Model of MYC-Mediated Repression
}

\author{
Michael D. Cole \\ Departments of Pharmacology and Genetics, Geisel School of Medicine at Dartmouth College, Lebanon, \\ New Hampshire 03756 \\ Correspondence: mcole@dartmouth.edu
}

MYC is one of the most frequently mutated and overexpressed genes in human cancer but the regulation of MYC expression and the ability of MYC protein to repress cellular genes (including itself) have remained mysterious. Recent genome-wide association studies show that many genetic polymorphisms associated with disease risk map to distal regulatory elements that regulate the MYC promoter through large chromatin loops. Cancer risk-associated single-nucleotide polymorphisms (SNPs) contain more potent enhancer activity, promoting higher MYC levels and a greater risk of disease. The MYC promoter is also subject to complex regulatory circuits and limits its own expression by a feedback loop. A model for MYC autoregulation is discussed which involves a signaling pathway between the PTEN (phosphatase and tensin homolog) tumor suppressor and repressive histone modifications laid down by the EZH2 methyltransferase.

$T_{\text {titise }}^{\text {he }}$ he MYC proto-oncogene family has been at the forefront of cancer biology for $>30 \mathrm{yr}$ but it keeps presenting surprises at every turn. MYC was the earliest proto-oncogene found to be responsive to growth factors and also subject to feedback autoregulation, which prompted numerous studies into the promoter sequences that mediate these responses. Although MYC responds to virtually every signaling pathway in every cell type, no clear picture has ever emerged as to the precise location of MYC regulatory elements. A plethora of transcription factors have been described as binding to or activating the proximal MYC promoter, often in transient assays in which the promoter is removed from its normal chromosomal context. These studies have been well documented (reviewed in Liu and Levens 2006) and will not be discussed in detail here. However, the picture of MYC regulation has become even murkier with knowledge of complete genome sequences and the advent of comprehensive genomics analyses of in vivo transcription factor binding sites and chromatin modifications. A curiosity of the MYC gene that was evident when the human genome was sequenced is that there are no known functional protein coding genes anywhere nearby, constituting what is often referred to as a "gene desert" (Fig. 1). This desert is huge $(\sim 3 \mathrm{Mb})$, and it occupies nearly the entirety of chromosomal band 8q24. MYC exists in a gene desert in all mammals, and this configuration extends to all vertebrates, although the desert gets smaller in more primitive species. Why does a small gene of only $5 \mathrm{~kb}$ occupy such a large space in the genome?

Editors: Chi V. Dang and Robert N. Eisenman

Additional Perspectives on MYC and the Pathway to Cancer available at www.perspectivesinmedicine.org

Copyright (C) 2014 Cold Spring Harbor Laboratory Press; all rights reserved; doi: 10.1101/cshperspect.a014316 Cite this article as Cold Spring Harb Perspect Med 2014;4:a014316 
M.D. Cole

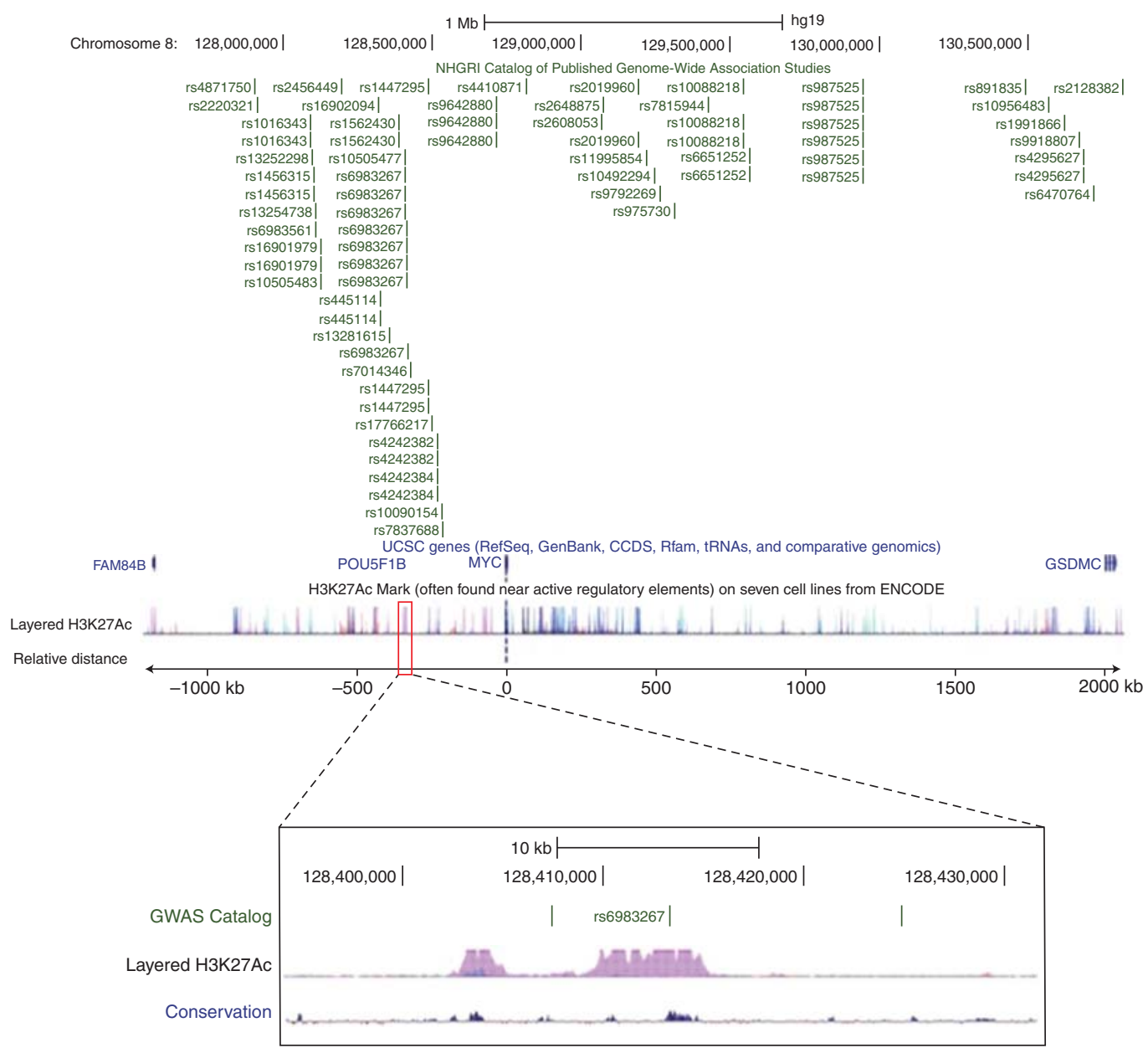

Figure 1. The MYC gene is in a huge "gene desert" on chromosomal band 8q24. The MYC gene maps to chromosomal band 8q24 and there are no known functional genes in the $1.2 \mathrm{Mb}$ region $5^{\prime}$ of the gene (left side) or the $2 \mathrm{Mb}$ region $3^{\prime}$ of the gene (right side). POU5F1B is likely a processed pseudogene, which has not been shown to make a functional protein, although the open reading frame is intact. Green numbers (top) are independent genome-wide association studies (GWAS) that identify numerous single-nucleotide polymorphisms (SNPs) within 8q24 that are linked to human disease. The H3K27ac track shows major peaks of histone $\mathrm{H} 3 \mathrm{~K} 27$ acetylation that are associated with active regulatory elements and these elements span the entire $3 \mathrm{Mb}$ region. References for each SNP and its disease association can be found on the GWAS track of the UCSC Genome Browser (Meyer et al. 2013). The expanded panel below shows local H3K27ac peaks near the rs6983267 SNP, which has been frequently associated with many types of cancer. The enhancer-linked modifications extend over a $4 \mathrm{~kb}$ region, part of which is highly conserved in mammalian evolution (bottom track). All data was extracted from the UCSC Genome Browser (Meyer et al. 2013).

\section{DISTAL REGULATION OF MYC: EARLY OBSERVATIONS}

The first hint that MYC could be regulated from a great linear genomic distance came from studies of chromosomal translocations and retrovi- ral integration sites in cancer cells. Although the first translocation breakpoints and retroviral integration sites mapped near the MYC promoter or even within the gene, it was soon discovered that some breakpoints and integration sites mapped far downstream from MYC 
$(150-300 \mathrm{~kb})$ in a region that was called PVT for plasmacytoma variant translocation (Cory et al. 1985). In mouse plasmacytomas and human Burkitt's lymphomas, an immunoglobulin light-chain gene is translocated onto chromosome 8, downstream from MYC, yet the MYC gene remains on chromosome 8 and all proximal regulatory sequences remain unperturbed. This same translocation breakpoint region is also a common site of retroviral integrations in mouse T-cell lymphomas (Graham et al. 1985), with very few integration sites in the intervening region between PVT1 and MYC. When possible, it was shown that only the translocated or virally targeted MYC allele was expressed, whereas the unaltered MYC allele was silenced by autoregulation (Nishikura et al. 1983; Lazo et al. 1990). These studies suggested that the MYC gene could be regulated in cis from a great distance, presumably through interactions between the MYC promoter and regulatory elements in the immunoglobulin gene or retrovirus, although it was not possible to show this directly at the time.

\section{MYC AND THE INHERITED PREDISPOSITION TO CANCER}

Sequencing of the human genome not only allowed the positions of all genes to be mapped but also revealed the enormous genetic variation between individuals. Single-nucleotide polymorphisms (SNPs) are found throughout the genome, with $>10^{6}$ DNA sequence variations between any two individuals. This variation allows genome-wide association studies (GWAS) to map linkage between all SNPs and any given trait, such as cancer risk. One of the first whole genome analyses identified an inherited sequence variant within chromosomal band $8 \mathrm{q} 24$ that was associated with prostate cancer risk (Amundadottir et al. 2006). Many subsequent GWAS publications have identified other SNPs linked to prostate, colon, breast, ovarian, and bladder cancers, as well as chronic lymphocytic leukemia and Hodgkin's lymphoma (Fig. 1). There are more cancer risk-associated SNPs in 8q24 than anywhere else in the genome. Cancer risk SNPs are usually reported in terms of an "odds ratio" (OR) (i.e., the ratio of cancer incidence between the higher risk and lower risk alleles). Most of the risk SNPs within $8 \mathrm{q} 24$ have odds ratios of $1.25-1.5$, although two of the prostate cancer SNPs have ORs of $\sim 2$, meaning that twice as many men with the risk allele get cancer compared with the other. Traits other than cancer have also been linked to $8 \mathrm{q} 24$, such as a risk of end-stage renal disease and type 2 diabetes (Fig. 1). Notably, the risk-associated SNPs map both $5^{\prime}$ and $3^{\prime}$ of MYC and span $\sim 2.5 \mathrm{Mb}$ of the surrounding $3 \mathrm{Mb}$ gene desert, with some SNPs mapping as far as $2 \mathrm{Mb}$ away. Given the distances, the majority of these SNPs are unlikely to be linked to one another but instead mark separate loci that impact on a specific disease. Another notable feature is that the SNPs are usually linked to one cancer type and not others. For example, rs13281615 is linked to breast cancer risk but not prostate or colon cancer (Easton et al. 2007), and rs9642880 is linked to bladder cancer and not any other cancer types (Kiemeney et al. 2008). However, there are some SNPs that can alter the risk for multiple cancer types such as rs6983267, which is associated with the risk of both prostate and colon cancer as well as others (Tomlinson et al. 2007; Yeager et al. 2007, 2008; Wokolorczyk et al. 2008; Curtin et al. 2009).

\section{MECHANISM OF DISTAL REGULATION}

The mapping of cancer risk SNPs hundreds of $\mathrm{kb}$ away from the small $5 \mathrm{~kb}$ MYC transcribed region immediately posed a conundrum. How could single base changes in a noncoding region of the chromosome have a significant impact on cancer risk? Insight into this came from a variety of clues. First, whole genome mapping of histone modifications finds innumerable elements with the characteristics of enhancers interspersed throughout noncoding regions, often far from any genes such as in 8q24 (Heintzman et al. 2007, 2009). Second, evolutionary studies identify well-conserved segments within distal noncoding regions, implying functional significance. Finally, there is growing evidence for distal regulatory interactions mediated by chromatin loops throughout the genome, perhaps best studied in mammals at the globin locus with the 
M.D. Cole

distal locus control region that regulates sequential gene expression during development (Tolhuis et al. 2002; Miele and Dekker 2008).

The first clear picture of how a distal SNP could regulate MYC expression came from studies of rs6983267, which maps $335 \mathrm{~kb}$ upstream of MYC. The rs6983267 SNP is either G (cancer risk) or $\mathrm{T}$ (lower risk), and it increases the risk of both colon and prostate cancer by $25 \%-50 \%$, depending on the inheritance of either one or two risk alleles. The worldwide allele distribution of rs6983267 is interesting because all individuals of African descent are homozygous for the risk allele $(\mathrm{G} / \mathrm{G})$, whereas European and Asian populations have a 1:1 allele ratio $(\mathrm{G} / \mathrm{T})$. GWAS of the African-American population finds that they have a higher risk of prostate cancer than European populations based on this single SNP (Gudmundsson et al. 2007). Genomic analysis showed that rs6983267 maps within a $1 \mathrm{~kb}$ segment that is highly conserved in mammalian evolution (Fig. 2). The cancer risk allele $(\mathrm{G})$ is found in all mammals, indicating that it is the ancestral allele, and the Tallele presumably emerged at some point in human evolu- tion. Most importantly, rs6983267 falls within a consensus motif for binding to the TCF-4/ LEF-1 transcription factor, the major mediator of Wnt signaling in the nucleus (Wright et al. 2010). Previous studies showed that MYC is induced by the WNT pathway and is a critical mediator of WNT signaling (He et al. 1998; van de Wetering et al. 2002). However, the specific regulatory element for $\mathrm{WNT} / \mathrm{TCF}-4$-mediated MYC induction had never been clearly defined. Chromatin immunoprecipitation (ChIP) experiments showed that both TCF-4 and its cofactor $\beta$-catenin bind to the rs6983267 SNP and binding is significantly stronger for the cancer risk allele (Wright et al. 2010). Furthermore, histone modifications associated with transcriptional enhancer activity are enriched on the risk allele, suggesting a more potent regulatory element (Wright et al. 2010). Direct proof of the allele-specific enhancer activity came from luciferase reporter assays that showed elevated TCF responsiveness for the risk allele (Pomerantz et al. 2009a; Tuupanen et al. 2009).

With knowledge that rs6983267 mapped to an evolutionarily conserved TCF-4-respon-

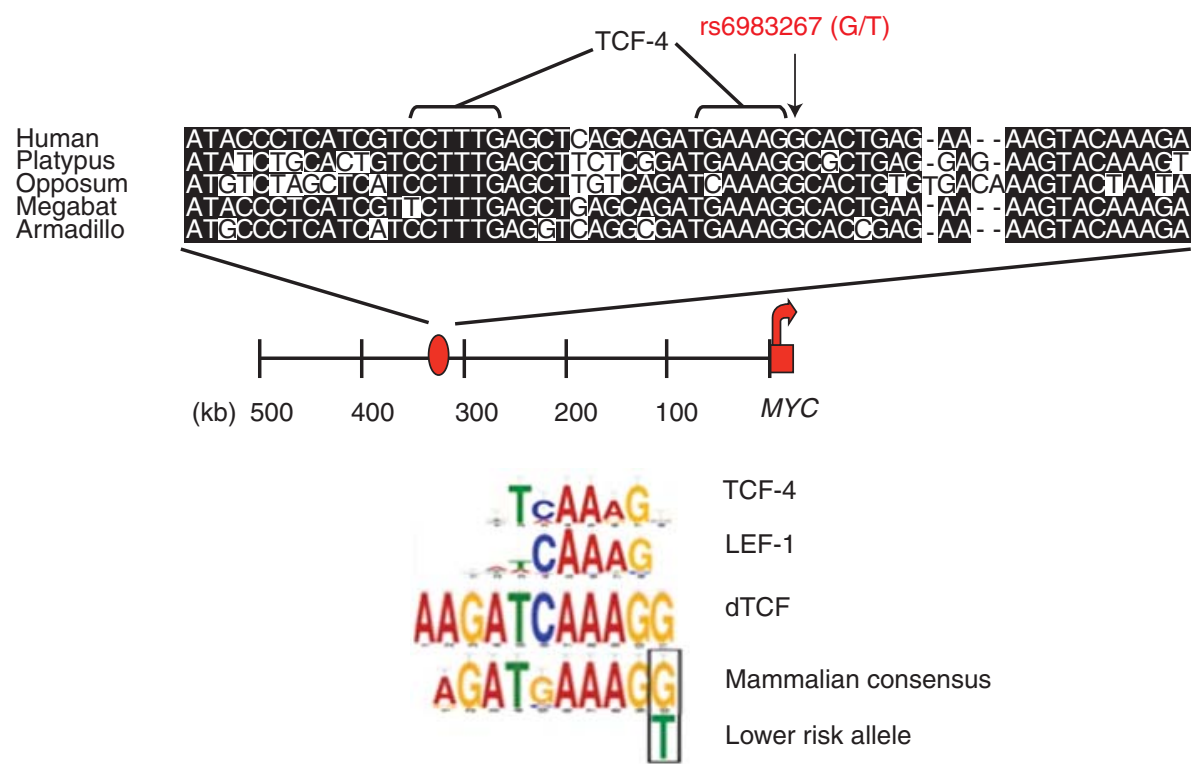

Figure 2. Evolutionary conservation of the rs6983267 SNP. The rs6983267 SNP is highly conserved among diverse mammals and associated with a consensus binding site for the TCF-4/LEF- 1 transcription factor. The cancer risk allele $(\mathrm{G})$ is found in all mammals, whereas the lower risk allele $(\mathrm{T})$ is only found in a fraction of the human population. (Figure based on Wright et al. 2010.) 
MYC Regulation, Repression, and Cancer

sive enhancer, the challenge was to determine whether MYC was the regulatory target even though the gene is $335 \mathrm{~kb}$ away. The obvious solution was a chromosomal loop linking the enhancer and the promoter. Chromosome looping can be detected using chromosome conformation capture (3C), which uses formaldehyde-fixed chromatin to lock chromosome loops into proximity (Dekker 2006). The chromosomal DNA is then digested with a restriction enzyme, extensively diluted, then treated with ligase. DNA fragments tethered together at the base of a loop ligate because of their fixed proximity, whereas DNA that is freely diffusing in unlinked complexes will not ligate because it is too dilute. Loops are analyzed by PCR with widely separated primers that detect the ligation of distal chromosomal sites. A systematic survey of the entire $350 \mathrm{~kb}$ region $5^{\prime}$ of the MYC gene revealed only a single prominent loop that connects the rs6983267 enhancer and the MYC promoter (Fig. 3) (Pomerantz et al. 2009a; Tuupanen et al. 2009; Wright et al. 2010). This finding provided direct evidence for a potential regulatory interaction between rs6983267 and MYC. Curiously, the rs6983267 enhancer also loops to the PVT1 promoter, albeit much more weakly (Wright et al. 2010). PVT1 is a noncod- ing RNA that initiates $30 \mathrm{~kb} 3^{\prime}$ of MYC, more distal to rs6983267. The significance of this will be discussed in more detail below.

What creates and maintains the rs6983267MYC chromatin loop? This is an active area of investigation for all nuclear architecture, and key questions remain unresolved. Two factors frequently associated with chromatin loops are the zinc-finger transcription factor CTCF and the chromosome pairing protein cohesin $(\operatorname{rad} 21)$ (reviewed in Wood et al. 2010). Both factors together or singly are found at the majority of chromatin loops, which represent novel functions for these proteins, and which were first associated with boundary elements and chromosome cohesion in $\mathrm{G}_{2} / \mathrm{M}$, respectively. Although CTCF has sequence-specific DNAbinding activity, cohesin has no direct DNAbinding activity but instead can hold DNA strands together in a "donut" structure. Depletion of either factor can disrupt chromosome looping. Consistent with the roles of these proteins in looping, both the rs6983267 enhancer and the MYC promoter have major peaks of CTCF and cohesin binding in ChIP-seq mapping experiments. Depletion of CTCF has a minimal effect on Myc expression in colon carcinoma cells, whereas depletion of cohesin re-

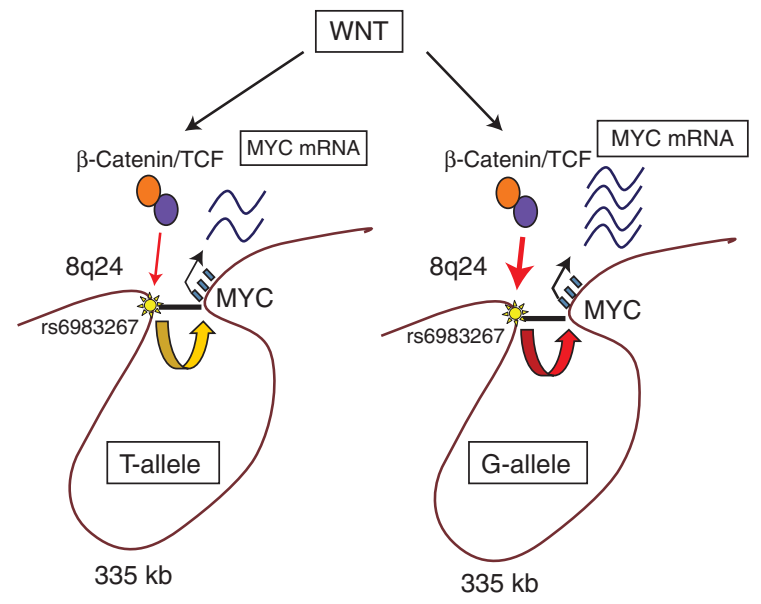

Figure 3. Model for the allele-specific cancer risk of rs6983267. The rs6983267 enhancer region forms a large chromatin loop $(335 \mathrm{~kb})$, which brings it into proximity with the MYC promoter. The cancer risk allele $(\mathrm{G}$, right) is a higher-affinity binding site for the TCF- $4 / \beta$-catenin transcription factor complex in the WNT signaling pathway, which is highly active in colon cancer cells owing to loss of the APC tumor suppressor. Higher-affinity TCF-4/ $\beta$-catenin binding promotes higher levels of MYC mRNA to increase cancer risk. 
M.D. Cole

duces MYC expression dramatically (J Wright, pers. comm.). In contrast to cohesin, the level of $\beta$-catenin/TCF-4 does not affect chromatin looping because cells with both high and low WNT pathway signaling have equally prominent loops (Wright et al. 2010). Furthermore, looping is not allele specific in colon cancer cells that are heterozygous for the rs6983267 SNP (Wright et al. 2010).

\section{MYC EXPRESSION AND CANCER RISK}

If MYC is the regulatory target of the rs6983267 enhancer, one would expect higher expression from the cancer risk allele because higher MYC levels are associated with cancer. However, studies of prostate cancer failed to show a clear association of different genotypes with MYC expression levels (Pomerantz et al. 2009b). Another approach was to study allele-specific MYC expression in a cell line heterozygous for the rs6983267 SNP as with the loop studies above. Fortuitously, DLD1 cells are heterozygous for both the rs6983267 SNP and a second SNP (rs4645953) in the MYC first intron, allowing studies of allele-specific MYC expression. Given the distance between rs6983267 and rs4645953 (335 kb), the chromosomal linkage between the SNPs was challenging to determine but 3C studies showed that the rs6983267 cancer risk allele $(\mathrm{G})$ is linked to the rs4645953(C) allele (Wright et al. 2010). Analysis of RNA levels by RT-PCR showed that the MYC allele linked to the cancer risk SNP is expressed at an approximately twofold higher level than the MYC allele linked to the lower risk SNP (Wright et al. 2010). This provided the first direct evidence for altered MYC expression caused by a cancer risk SNP.

An important question concerning the rs6983267 SNP is when in development does it influence cancer risk. In contrast to its frequent association with cancer, rs6983267 has never been scored as an eQTL (expression quantitative trait locus), which are SNPs that influence gene expression at a genome-wide level. Given the importance of MYC in development, rs6983267 might have been scored as an eQTL if it had any significant impact on a human trait other than cancer or on broad gene expression.
Even though the G allele is the ancestral form in mammals and the $\mathrm{T}$ allele has a significantly lower affinity for TCF- $4 / \beta$-catenin binding (Wright et al. 2010), the lack of an influence as an eQTL suggests that there must be redundancy or compensation in MYC regulatory elements so that the TCF-4 binding affinity difference has no phenotype. On the other hand, a recent mouse model underscores the importance of the rs6983267 enhancer region in colon cancer (Sur et al. 2012). Deletion of the enhancer in the mouse germ line had a minor effect on MYC expression but no effect on development, consistent with the lack of eQTL influence in humans. In contrast, crossing the rs6983267 enhancer-deficient line to the "APCmin" mouse, which harbors a germ line mutation in the APC tumor suppressor, leads to a dramatic reduction in intestinal tumor development (Sur et al. 2012). These observations provide a graphic demonstration of the essential role of the rs6983267 enhancer as a "target" of the excessive Wnt signaling found in colon cancers. In combination with the human GWAS observations, it would appear that rs6983267 only becomes a significant factor when there are mutations in the Wnt pathway in colon cancer cells.

Interestingly, a second high-affinity TCF-4/ LEF-1 binding site has been mapped $1.4 \mathrm{~kb}$ downstream from MYC, but this site is not polymorphic in the population or associated with any disease risk (Konsavage et al. 2012). Surprisingly, genetic knockout of this element increases cell proliferation and MYC levels, exactly the opposite of the rs6983267 enhancer. The higher levels of MYC are associated with increased numbers of proliferative cells in the colon. Thus, TCF-4 binding sites can act as both activators and repressors of MYC expression, and what distinguishes this differential response is unknown.

\section{MYC AND PROSTATE CANCER RISK}

The most common cancer risk linked to 8q24 predisposes to prostate cancer. At least 10 SNPs within $8 \mathrm{q} 24$ have been linked to prostate cancer risk, and these SNPs span a region 600-200 kb upstream of MYC (Fig. 1). The majority of these 
SNPs are linked only to prostate cancer and no other cancer types. MYC expression is high in prostate cancer (Gurel et al. 2008), and somatic mutations in the form of MYC gene amplifications are common (Jenkins et al. 1997), so it is not surprising that MYC could also be linked to inherited cancer risk. Based on the rs6983267 paradigm, these SNPs are predicted to be linked to a prostate-specific regulatory element, and the great distance between the different SNPs suggests that there are multiple independent elements. However, a molecular mechanism has been proposed for only two SNPs linked to prostate cancer risk: rs378854, which is in linkage disequilibrium (LD) with the GWAS SNP rs620861 (Meyer et al. 2011), and also the independent SNP rs1456315 (Chung et al. 2011). Surprisingly, rs378854 was suggested to not affect MYC expression at all (Meyer et al. 2011). Instead, a large chromatin loop was mapped between rs378854 and the promoter of PVT1, which is a long noncoding RNA (lncRNA) on the $3^{\prime}$ side of MYC. The minor protective allele of rs378854 binds avidly to the transcription factor YY1 and forms a repressive interaction with the PVT1 promoter, leading to slightly lower expression (Meyer et al. 2011). The common prostate cancer risk allele of rs378854 binds YY1 more weakly and fails to repress PVT1. Similarly, the GWAS SNP rs1456315 was shown to be linked to two other SNPs that are found within another lncRNA (PRNCR1) transcribed from a distal region $5^{\prime}$ of MYC. The function of the PVT1 and PRNCR1 lncRNAs remains unclear but there are reports of roles in cancer cell growth (reviewed in Huppi et al. 2012).

Although the majority of cancer risk SNPs map to the $5^{\prime}$ gene desert upstream of MYC, the risk for some cancers and other diseases maps within the equally large gene desert $3^{\prime}$ of MYC. Notably, risk SNPs for Hodgkin's lymphoma, ovarian cancer, inflammatory bowel disease, and end-stage renal disease map over a $500 \mathrm{~kb}$ region $3^{\prime}$ of MYC (Fig. 1). Two of the risk SNPs fall within the transcribed region for the PVT1 lncRNA, although it is not known whether this is functionally relevant. As with the risk associations that map $5^{\prime}$ of MYC, the great distance between individual SNPs suggests that independent regulatory elements are contributing to the disease. Moreover, as with most of the $5^{\prime}$ SNPs, it is not clear which $3^{\prime}$ SNPs are directly responsible for the disease risk versus which are simply linked to the causal SNP and score in GWAS owing to tight linkage.

\section{ENHANCERS, LOOPS, AND MORE ENHANCERS}

One of the remarkable features of the 8q24 gene desert is the huge number of DNA elements with characteristics of enhancers, which are spread over a vast linear distance along the chromosome. If strong peaks of H3K27 acetylation are used as an indicator of potential enhancers, there are more than 30 enhancers in the $1 \mathrm{Mb}$ region $5^{\prime}$ from $\mathrm{MYC}$ in the multiple cell types analyzed by ChIP-seq as part of the ENCODE project (Fig. 1). If smaller peaks of $\mathrm{H} 3 \mathrm{~K} 27 \mathrm{ac}$ mark functional elements, there could be double that number of enhancers. Most of these sequences are conserved in evolution among mammals, providing further evidence of functional significance. The gene desert $3^{\prime}$ of MYC is even larger than the $5^{\prime}$ region, and it contains similar numbers of enhancer-like elements. Do all of these potential elements contribute to the regulation of a relatively simple gene like MYC? Are all of these enhancer-like elements functional in various cell types and at different stages of development? The answer may take years to resolve but could stem from the requirement for MYC to respond to a multitude of different signaling networks. Furthermore, because any given cell has multiple signaling networks that impact on MYC expression, many regulatory elements will be simultaneously primed to respond. Continued studies of the vast regulatory region surrounding MYC should help resolve these important questions.

\section{MYC-MEDIATED REPRESSION}

The first MYC-regulated gene ever described was MYC itself, in which overexpression of MYC after chromosomal translocation leads to suppression of MYC expression from the nor- 
M.D. Cole

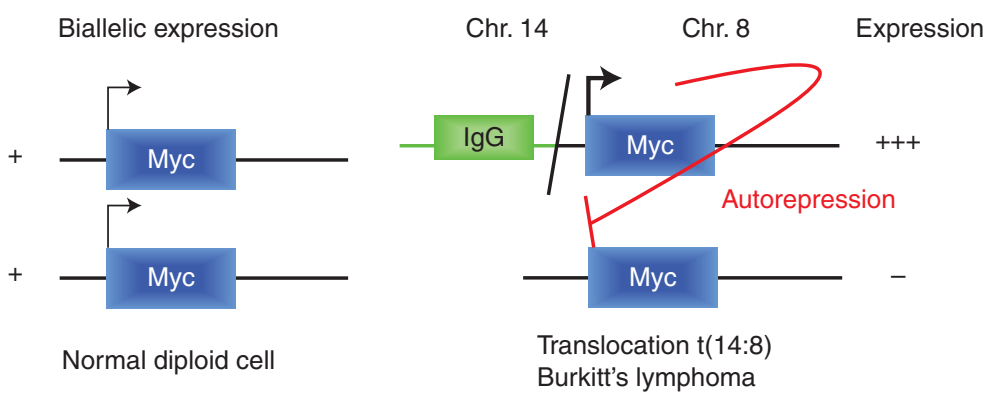

Figure 4. Autorepression of MYC expression. In normal cells, both MYC alleles are expressed at equal levels and subject to tight regulation by diverse signaling pathways. In Burkitt's lymphoma, one MYC allele is translocated to the immunoglobulin heavy chain region where a strong enhancer promotes high levels of expression. The remaining allele is silenced by a feedback loop, which keeps MYC levels in check in normal cells. Chr., chromosome.

mal, nontranslocated allele (Fig. 4) (Nishikura et al. 1983). This phenomenon has usually been attributed to a feedback autoregulatory loop that limits the amount of MYC expression when levels are high, because high levels of MYC potentiate cancer. Despite considerable effort, the mechanism of MYC autoregulation has never been explained. Notably, only the basal MYC promoter seems to be required for autoregulation (Facchini et al. 1997), suggesting that no specific transcription factors are involved. Cancer cells often lose the ability to autoregulate MYC, presumably leading to higher constitutive levels (Grignani et al. 1990; Penn et al. 1990). Autoregulation of MYC is not the only MYC-repressed gene, because gene expression profiling finds nearly an equal number of genes repressed by MYC as activated (Cowling et al. 2006). Furthermore, MYC-mediated repression is dependent on the MB2 domain (Bush et al. 1998), an extraordinarily well-conserved motif within the MYC protein transactivation domain. The MB2 domain has been shown to be required to recruit several transcriptional cofactors to DNA (reviewed in Cowling and Cole 2006) and for a large fraction of both MYC-activated and MYC-repressed genes (Cowling et al. 2006).

Two mechanisms of MYC-mediated repression have been proposed. The first involves a zinc-finger transcription factor called Miz-1, which can bind to the INR element within basal promoters (reviewed in Adhikary and Ei- lers 2005). Binding of Miz-1 to the INR leads to transcriptional activation, whereas subsequent binding of MYC to Miz-1 represses activation or yields a net repression. This mechanism has been shown to be active for repression of the p15 and p21 tumor suppressors and for the BCL2 antiapoptotic gene among others (Gartel et al. 2001; Seoane et al. 2001; Staller et al. 2001; Patel and McMahon 2007). An alternative mechanism of repression was suggested by studies in the fruit fly Drosophila melanogaster, which implicated polycomb repression complexes (PRC1) both in MYC autoregulation and in general MYC-mediated repression (Goodliffe et al. 2005). A second polycomb repressive complex (PRC2) exists in both mammals and fruit flies, and PRC2 can mediate gene repression by methylation of the amino-terminal tails of histone $\mathrm{H} 3$ on lysine 27 (H3K27me3) (reviewed in Bracken and Helin 2009). Histone methylation is performed by the methyltransferase enzyme EZH2 (or Enhancer of Zeste in fruit flies), which in turn can lead to compacted chromatin and/or recruitment of PRC1. The EZH2 gene is frequently amplified or overexpressed in human cancer (Bracken et al. 2003).

\section{HISTONE METHYLATION AT MYC-REPRESSED GENES}

Because repression by the PRC2 complex containing the EZH2 methyltransferase occurs mainly through H3K27me3, we used ChIP on 
MYC Regulation, Repression, and Cancer

several well-established MYC-repressed genes in two different cell systems to show a dramatic induction of $\mathrm{H} 3 \mathrm{~K} 27 \mathrm{me} 3$ after MYC overexpression, including at the MYC promoter itself (Kaur and Cole 2013). Induction of H3K27me3 was accompanied by enhanced binding of EZH2 and the PRC2 complex, which is known to bind to its own modification sites. Importantly, depletion of EZH2 by siRNA eliminated both H3K27me3 accumulation and MYC-mediated repression, arguing that histone modification is required for repression and autoregulation.

\section{REGULATION OF PRC2 ACTIVITY BY PHOSPHORYLATION}

How could MYC control EZH2 and histone modification? The clue to this came from a previous study of EZH2 that showed that its activity was regulated by phosphorylation of serine 21 (Cha et al. 2005). Phosphorylated EZH2 has reduced histone methyltransferase activity and a reduced ability to interact with $\mathrm{H} 3$. Using phosphospecific antibodies, assays of EZH2 showed that MYC overexpression leads to a major reduction in phosphorylation in two different cell systems (Kaur and Cole 2013). The kinase reported to phosphorylate S21 is Akt, and we showed that MYC could repress active, phosphorylated Akt in parallel with reduced EZH2 phosphorylation. Reduced Akt activity and reduced EZH2 phosphorylation would lead to enhanced EZH2 activity.

\section{MYC ACTIVATES PTEN}

The tumor suppressor PTEN ( phosphatase and tensin homolog) regulates the PI3 kinase pathway and AKT phosphorylation, and PTEN is frequently inactivated in human cancer (Chalhoub and Baker 2009). PTEN was previously shown to be a direct MYC target gene based on studies of gene expression in a MYC-mediated chicken lymphoma model system and on ChIP of MYC protein to a consensus MYC/ MAX binding site in the PTEN promoter (Neiman et al. 2001; Fernandez et al. 2003). Consistent with these previous studies, PTEN mRNA was shown to be transcriptionally induced by
MYC in two cell systems showing MYC-mediated repression and autoregulation (Kaur and Cole 2013).

If the transcriptional induction of PTEN is required for MYC-mediated repression and autoregulation, then depleting PTEN would be expected to alter repression. Indeed, depletion of PTEN levels using siRNA led to a complete reversal of MYC repression and autoregulation. Furthermore, derepression was accompanied by loss of the H3K27me3 modification that results from EZH2 activation, further validating the PTEN-AKT-EZH2 pathway (Kaur and Cole 2013).

\section{EZH2 ACTIVATION IS SUFFICIENT TO ACCOUNT FOR MYC-MEDIATED REPRESSION}

Having established a critical regulatory mechanism linking PTEN transcriptional induction and MYC-mediated repression, it was important to establish the breadth of this pathway in gene regulation. To this end, a mutant EZH2 was created that lacked the AKT phosphorylation site (EZH2/S21A) and hence cannot be inactivated by phosphorylation. Wild-type $\mathrm{EZH} 2$ and the EZH2/S21A mutant were stably expressed in $\mathrm{MYC}^{-/-}$rat fibroblasts to analyze EZH2 activity in the absence of any influence of endogenous MYC. Consistent with the hypothesis that EZH2 activity controls global H3 methylation, we found that total cellular $\mathrm{H} 3 \mathrm{~K} 27 \mathrm{me} 3$ levels were dramatically elevated by both MYC and EZH2/S21A expression (Kaur and Cole 2013). Previous studies showed that MYC can increase total cellular levels of the activating histone modifications, acetylated H3 and acetylated H4 (Knoepfler et al. 2006), but this experiment showed a simultaneous increase in the repressive $\mathrm{H} 3 \mathrm{~K} 27 \mathrm{me} 3$ modification. Because the EZH2/S21A mutant can completely recapitulate the effect of MYC on H3K27me3 levels, this is compelling evidence that MYC acts through reduced phosphorylation of EZH2.

In addition to global H3K27me3 levels, the $\mathrm{EZH} 2 / \mathrm{S} 21 \mathrm{~A}$ mutant alone was able to repress the MYC promoter and other MYC-repressed genes, which showed the same local elevation 
M.D. Cole

in $\mathrm{H} 3 \mathrm{~K} 27 \mathrm{me} 3$ modification as in the response to MYC overexpression (Kaur and Cole 2013). Interestingly, increased $\mathrm{H} 3 \mathrm{~K} 27 \mathrm{me} 3$ levels were accompanied by loss of $\mathrm{H} 3 \mathrm{~K} 4 \mathrm{me} 3$, a modification linked to active promoters. The critical question was how much of MYC-mediated repression could be accounted for by EZH2 activity alone. Microarray experiments were conducted to assess the full extent of gene expression changes in response to MYC or the EZH2/S21A mutant. Remarkably, EZH2/S21A expression alone was sufficient to repress $45 \%$ of all genes repressed by MYC (Kaur and Cole 2013). Thus, the PTENAKT-EZH2 pathway can account for nearly half of all MYC-mediated repression.

\section{IMPLICATIONS OF MYC-MEDIATED GENE REPRESSION}

The combined data from the studies outlined above lead to a novel model for MYC-mediated repression (Fig. 5) that goes a long way toward resolving several mysteries that have haunted the MYC field since the beginning, especially the critical homeostatic autoregulation (Kaur and Cole 2013). First, repression has been consistently found to be dependent on MB2, the same domain usually linked to transactivation (Cowling and Cole 2006). This is explained by the dependence for PTEN transcriptional activation, which is dependent on MB2. Second, the model can explain why cancer cells often lose MYC autoregulation, because loss of the PTEN tumor suppressor and activation of the $\mathrm{PI} 3 \mathrm{~K} / \mathrm{AKT}$ pathway are common genetic lesions in tumor cells (Chalhoub and Baker 2009). Third, it explains why direct MYC binding is not necessary for repression, because repression is indirectly mediated by PTEN/ PRC2/EZH2 activation. Finally, it explains why no specific promoter element mediating autoregulation could be mapped in the MYC promoter (Facchini et al. 1997) and no consensus site has been linked to repression in general.
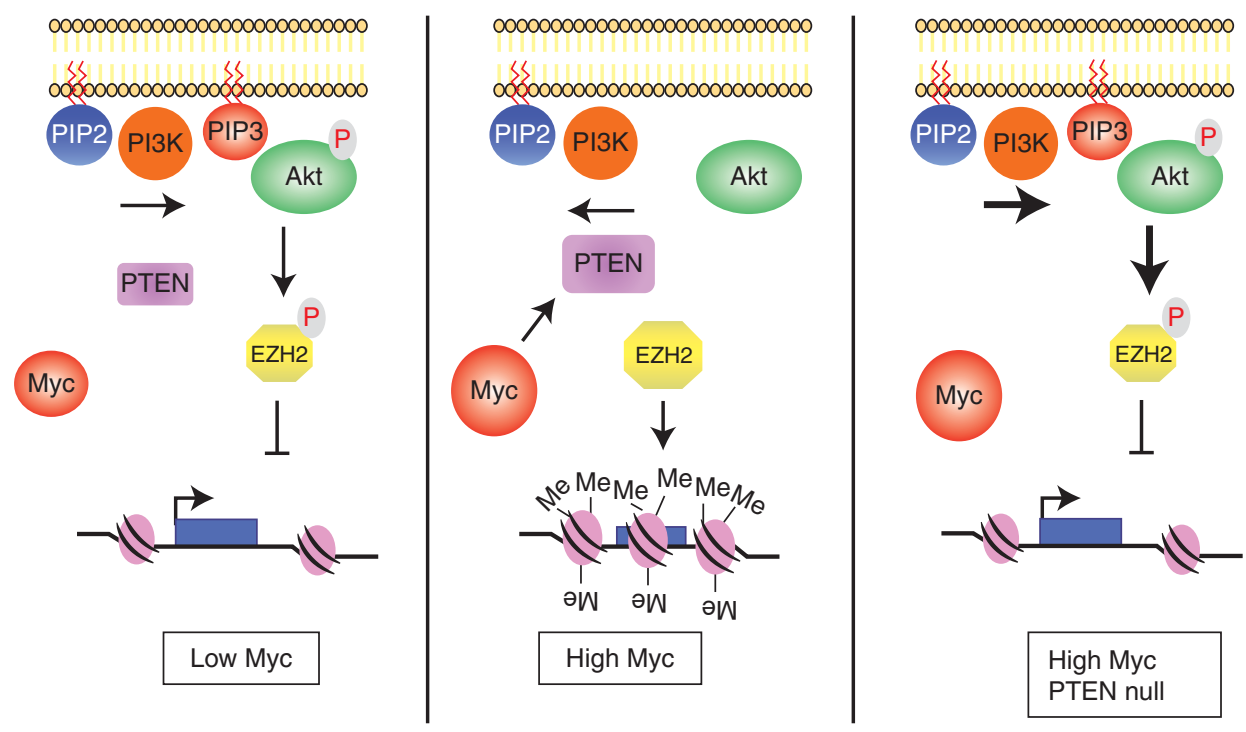

Figure 5. Model for MYC autorepression and general cellular gene repression by histone modification. In normal cells (left), MYC levels are low and EZH2 histone methyltransferase activity is suppressed by AKT phosphorylation. In cells with high MYC (center), the PTEN tumor suppressor is transcriptionally activated by MYC, leading to suppression of the PI3 kinase pathway and activation of EZH2 activity. Higher levels of EZH2 promote increased H3K27 methylation, which is necessary and sufficient to mediate MYC autorepression and cellular gene repression. In cancer cells, which genetically lose the PTEN tumor suppression (right) or which might have mutations in the PI3 kinase pathway, high levels of AKT activity continually suppress EZH2 activity and the accumulation of H3K27me3, preventing MYC autoregulation. (Figure based on data from Kaur and Cole 2013). 
MYC Regulation, Repression, and Cancer

Our model explains these observations in part because histone modifications can affect global gene expression and may not require specific promoter elements.

It is interesting to point out additional dynamics of this Myc repressive pathway in normal versus cancer cells. In normal cells, induction of Myc by growth factors would induce PTEN and subsequently enhance EZH2 activity and $\mathrm{H} 3 \mathrm{~K} 27 \mathrm{me} 3$ beyond any increase in EZH2 mRNA level. It is also interesting to consider that inactivation of AKT by PTEN could lead to decreased levels of Myc through GSK3b stimulation of T58 phosphorylation (Sears 2004). In contrast, loss of the PTEN tumor suppressor or activation of PI3K in cancer cells would lead to constitutive suppression of EZH2 and reduced genome-wide H3K27me3 (Fig. 5). On the other hand, EZH2 is frequently overexpressed and/or genetically amplified on cancer (Bracken et al. 2003), which would tend to restore levels of $\mathrm{H} 3 \mathrm{~K} 27 \mathrm{me} 3$ even in the face of constitutive phosphorylation and suppression by AKT.

The biggest challenge of this new model of MYC-mediated repression is to resolve how some genes are repressed by activation of EZH2 activity and increased levels of $\mathrm{H} 3 \mathrm{~K} 27 \mathrm{me} 3$, whereas an equal number of genes are activated. In Drosophila, polycomb complexes can be targeted to specific promoters by so-called polycomb response elements (PREs) (Muller and Kassis 2006). However, similar regulatory elements have not been convincingly identified in mammalian cells (Simon and Kingston 2009). There are at least two possible hypotheses that could account for selective repression of genes, especially the extreme variability between different cell types. One hypothesis is that increasing EZH2 activity nucleates H3K27me3 modifications through weak interactions with numerous transcription factors, and the PRC2 complex binds to its own modification sites (Hansen et al. 2008), giving a positive-feedback loop to spread repression locally, a well-known feature of polycomb complexes. There could be a highly variable balance between active and repressive chromatin at different genes and in different cells, explaining the extreme variability of MYC-mediated repression. A second hypothesis is that noncoding RNAs target PRC2 complexes to some promoters and not others (reviewed in Smith and Shilatifard 2010), which could also be enhanced by a positive-feedback loop. These are only two of many other possible mechanisms, and further studies will be required to resolve this question.

\section{FUTURE PROSPECTS}

Studies of MYC have revealed some of the most fundamental mechanisms associated with the development of cancer. The studies described above provide new insight into the role of MYC in cancer, from inherited genetic predisposition to gene repression and autoregulation. The level of MYC protein in normal cells is tightly controlled by transcription, and both distal regulatory elements and localized repression are major contributing factors. Continuing studies of MYC will undoubtedly reveal even more novel insights into cancer.

\section{ACKNOWLEDGMENTS}

Research from the author's laboratory described in this article is supported by grants from the National Institutes of Health National Cancer Institute. I thank Asaf Wyszynski for help with the preparation of figures.

\section{REFERENCES}

Adhikary S, Eilers M. 2005. Transcriptional regulation and transformation by Myc proteins. Nat Rev Mol Cell Biol 6: 635-645.

Amundadottir LT, Sulem P, Gudmundsson J, Helgason A, Baker A, Agnarsson BA, Sigurdsson A, Benediktsdottir KR, Cazier JB, Sainz J, et al. 2006. A common variant associated with prostate cancer in European and African populations. Nat Genet 38: 652-658.

Bracken AP, Helin K. 2009. Polycomb group proteins: Navigators of lineage pathways led astray in cancer. Nat Rev Cancer 9: 773-784.

Bracken AP, Pasini D, Capra M, Prosperini E, Colli E, Helin K. 2003. EZH2 is downstream of the pRB-E2F pathway, essential for proliferation and amplified in cancer. EMBO J 22: 5323-5335.

Bush A, Mateyak M, Dugan K, Obaya A, Adachi S, Sedivy J, Cole M. 1998. c-myc null cells misregulate cad and gadd45 but not other proposed c-Myc targets. Genes Dev 12: 3797-3802. 
M.D. Cole

Cha TL, Zhou BP, Xia W, Wu Y, Yang CC, Chen CT, Ping B, Otte AP, Hung MC. 2005. Akt-mediated phosphorylation of EZH2 suppresses methylation of lysine 27 in histone H3. Science 310: 306-310.

Chalhoub N, Baker SJ. 2009. PTEN and the PI3-kinase pathway in cancer. Annu Rev Pathol 4: 127-150.

Chung S, Nakagawa H, Uemura M, Piao L, Ashikawa K, Hosono N, Takata R, Akamatsu S, Kawaguchi T, Morizono T, et al. 2011. Association of a novel long non-coding RNA in 8q24 with prostate cancer susceptibility. Cancer Sci 102: 245-252.

Cory S, Graham M, Webb E, Corcoran L, Adams JM. 1985. Variant $(6 ; 15)$ translocations in murine plasmacytomas involve a chromosome 15 locus at least $72 \mathrm{~kb}$ from the c-myc oncogene. EMBO J 4: 675-681.

Cowling VH, Cole MD. 2006. Mechanism of transcriptional activation by the Myc oncoproteins. Semin Cancer Biol 16: $242-252$.

Cowling VH, Chandriani S, Whitfield ML, Cole MD. 2006 A conserved Myc protein domain, MBIV, regulates DNA binding, apoptosis, transformation, and $\mathrm{G}_{2}$ arrest. $\mathrm{Mol}$ Cell Biol 26: 4226-4239.

Curtin K, Lin WY, George R, Katory M, Shorto J, CannonAlbright LA, Bishop DT, Cox A, Camp NJ. 2009. Meta association of colorectal cancer confirms risk alleles at 8q24 and 18q21. Cancer Epidemiol Biomarkers Prev 18: $616-621$.

Dekker J. 2006. The three "C"s of chromosome conformation capture: Controls, controls, controls. Nat Methods 3: $17-21$.

Easton DF, Pooley KA, Dunning AM, Pharoah PD, Thompson D, Ballinger DG, Struewing JP, Morrison J, Field H, Luben R, et al. 2007. Genome-wide association study identifies novel breast cancer susceptibility loci. Nature 447: 1087-1093.

Facchini LM, Chen S, Marhin WW, Lear JN, Penn LZ. 1997. The Myc negative autoregulation mechanism requires Myc-Max association and involves the c-Myc P2 minimal. Mol Cell Biol 17: 100-114.

Fernandez PC, Frank SR, Wang L, Schroeder M, Liu S, Greene J, Cocito A, Amati B. 2003. Genomic targets of the human c-Myc protein. Genes Dev 17: 1115-1129.

Gartel AL, Ye X, Goufman E, Shianov P, Hay N, Najmabadi F, Tyner AL. 2001. Myc represses the $\mathrm{p} 21^{\mathrm{WAF} 1 / \mathrm{CIP} 1}$ promoter and interacts with Sp1/Sp3. Proc Natl Acad Sci 98: 45104515.

Goodliffe JM, Wieschaus E, Cole MD. 2005. Polycomb mediates Myc autorepression and its transcriptional contro of many loci in Drosophila. Genes Dev 19: 2941-2946.

Graham M, Adams JM, Cory S. 1985. Murine T lymphomas with retroviral inserts in the chromosomal 15 locus for plasmacytoma variant translocations. Nature 314: 740743.

Grignani F, Lombardi L, Inghirami G, Sternas L, Cechova K, Dalla-Favera R. 1990. Negative autoregulation of c-myc gene expression is inactivated in transformed cells. EMBO J 9: 3913-3922.

Gudmundsson J, Sulem P, Manolescu A, Amundadottir LT, Gudbjartsson D, Helgason A, Rafnar T, Bergthorsson JT, Agnarsson BA, Baker A, et al. 2007. Genome-wide asso- ciation study identifies a second prostate cancer susceptibility variant at 8q24. Nat Genet 39: 631-637.

Gurel B, Iwata T, Koh CM, Jenkins RB, Lan F, Dang CV, Hicks JL, Morgan J, Cornish TC, Sutcliffe S, et al. 2008. Nuclear MYC protein overexpression is an early alteration in human prostate carcinogenesis. Mod Pathol 21: $1156-1167$.

Hansen KH, Bracken AP, Pasini D, Dietrich N, Gehani SS, Monrad A, Rappsilber J, Lerdrup M, Helin K. 2008. A model for transmission of the H3K27me3 epigenetic mark. Nat Cell Biol 10: 1291-1300.

He TC, Sparks AB, Rago C, Hermeking H, Zawel L, da Costa LT, Morin PJ, Vogelstein B, Kinzler KW. 1998. Identification of c-MYC as a target of the APC pathway. Science 281: $1509-1512$.

Heintzman ND, Stuart RK, Hon G, Fu Y, Ching CW, Hawkins RD, Barrera LO, Van Calcar S, Qu C, Ching KA, et al. 2007. Distinct and predictive chromatin signatures of transcriptional promoters and enhancers in the human genome. Nat Genet 39: 311-318.

Heintzman ND, Hon GC, Hawkins RD, Kheradpour P, Stark A, Harp LF, Ye Z, Lee LK, Stuart RK, Ching CW, et al. 2009. Histone modifications at human enhancers reflect global cell-type-specific gene expression. Nature 459: $108-112$.

Huppi K, Pitt JJ, Wahlberg BM, Caplen NJ. 2012. The 8q24 gene desert: An oasis of non-coding transcriptional activity. Front Genet 3: 69.

Jenkins RB, Qian J, Lieber MM, Bostwick DG. 1997. Detection of c-myc oncogene amplification and chromosomal anomalies in metastatic prostatic carcinoma by fluorescence in situ hybridization. Cancer Res 57: 524-531.

Kaur M, Cole MD. 2013. MYC acts via the PTEN tumor suppressor to elicit autoregulation and genome-wide gene repression by activation of the Ezh2 methyltransferase. Cancer Res 73: 695-705.

Kiemeney LA, Thorlacius S, Sulem P, Geller F, Aben KK, Stacey SN, Gudmundsson J, Jakobsdottir M, Bergthorsson JT, Sigurdsson A, et al. 2008. Sequence variant on 8 q24 confers susceptibility to urinary bladder cancer. Nat Genet 40: 1307-1312.

Knoepfler PS, Zhang XY, Cheng PF, Gafken PR, McMahon SB, Eisenman RN. 2006. Myc influences global chromatin structure. EMBO J 25: 2723-2734.

Konsavage WM Jr, Jin G, Yochum GS. 2012. The Myc 3' Wnt-responsive element regulates homeostasis and regeneration in the mouse intestinal tract. Mol Cell Biol 32: 3891-3902.

Lazo PA, Lee JS, Tsichlis PN. 1990. Long-distance activation of the Myc protooncogene by provirus insertion in Mlvi1 or Mlvi-4 in rat T-cell lymphomas. Proc Natl Acad Sci 87: $170-173$.

Liu J, Levens D. 2006. Making myc. Curr Top Microbiol Immunol 302: 1-32.

Meyer KB, Maia AT, O’Reilly M, Ghoussaini M, Prathalingam R, Porter-Gill P, Ambs S, Prokunina-Olsson L, Carroll J, Ponder BA. 2011. A functional variant at a prostate cancer predisposition locus at 8q24 is associated with PVT1 expression. PLoS Genet 7: e1002165.

Meyer LR, Zweig AS, Hinrichs AS, Karolchik D, Kuhn RM, Wong M, Sloan CA, Rosenbloom KR, Roe G, Rhead B, et 
al. 2013. The UCSC Genome Browser database: Extensions and updates 2013. Nucleic Acids Res 41: D64-D69.

Miele A, Dekker J. 2008. Long-range chromosomal interactions and gene regulation. Mol Biosyst 4: 1046-1057.

Muller J, Kassis JA. 2006. Polycomb response elements and targeting of Polycomb group proteins in Drosophila. Curr Opin Genet Dev 16: 476-484.

Neiman PE, Ruddell A, Jasoni C, Loring G, Thomas SJ, Brandvold KA, Lee R, Burnside J, Delrow J. 2001. Analysis of gene expression during $m y c$ oncogene-induced lymphomagenesis in the bursa of Fabricius. Proc Natl Acad Sci 98: 6378-6383.

Nishikura K, ar-Rushdi A, Erikson J, Watt R, Rovera G, Croce CM. 1983. Differential expression of the normal and of the translocated human c-myc oncogenes in B cells. Proc Natl Acad Sci 80: 4822-4826.

Patel JH, McMahon SB. 2007. BCL2 is a downstream effector of MIZ-1 essential for blocking c-MYC-induced apoptosis. J Biol Chem 282: 5-13.

Penn LJZ, Brooks MW, Laufer EM, Littlewood TD, Morgenstern JP, Evan GI, Lee WMF, Land H. 1990. Domains of human c-Myc protein required for autosuppression and cooperation with ras oncogenes are overlapping. Mol Cell Biol 10: 4961-4966.

Pomerantz MM, Ahmadiyeh N, Jia L, Herman P, Verzi MP, Doddapaneni H, Beckwith CA, Chan JA, Hills A, Davis M, et al. 2009a. The 8q24 cancer risk variant rs6983267 shows long-range interaction with MYC in colorectal cancer. Nat Genet 41: 882-884.

Pomerantz MM, Beckwith CA, Regan MM, Wyman SK, Petrovics G, Chen Y, Hawksworth DJ, Schumacher FR, Mucci L, Penney KL, et al. 2009b. Evaluation of the 8q24 prostate cancer risk locus and MYC expression. Cancer Res 69: 5568-5574.

Sears RC. 2004. The life cycle of C-myc: From synthesis to degradation. Cell Cycle 3: 1133-1137.

Seoane J, Pouponnot C, Staller P, Schader M, Eilers M, Massague J. 2001. TGF $\beta$ influences Myc, Miz-1, and Smad to control the CDK inhibitor pp15 ${ }^{\text {INK4b }}$. Nat Cell Biol 3: 400-408.

Simon JA, Kingston RE. 2009. Mechanisms of polycomb gene silencing: Knowns and unknowns. Nat Rev Mol Cell Biol 10: 697-708.

Smith E, Shilatifard A. 2010. The chromatin signaling pathway: Diverse mechanisms of recruitment of histonemodifying enzymes and varied biological outcomes. Mol Cell 40: 689-701.

Staller P, Peukert K, Kiermaier A, Seoane J, Lukas J, Karsunky H, Moroy T, Bartek J, Massague J, Hanel F, et al.
2001. Repression of $\mathrm{p} 15^{\mathrm{INK} 4 \mathrm{~b}}$ expression by Myc through association with Miz-1. Nat Cell Biol 3: 392-399.

Sur IK, Hallikas O, Vaharautio A, Yan J, Turunen M, Enge M, Taipale M, Karhu A, Aaltonen LA, Taipale J. 2012. Mice lacking a Myc enhancer that includes human SNP rs6983267 are resistant to intestinal tumors. Science 338: $1360-1363$.

Tolhuis B, Palstra RJ, Splinter E, Grosveld F, de Laat W. 2002. Looping and interaction between hypersensitive sites in the active $\beta$-globin locus. Mol Cell 10: 1453-1465.

Tomlinson I, Webb E, Carvajal-Carmona L, Broderick P, Kemp Z, Spain S, Penegar S, Chandler I, Gorman M, Wood W, et al. 2007. A genome-wide association scan of tag SNPs identifies a susceptibility variant for colorectal cancer at 8q24.21. Nat Genet 39: 984-988.

Tuupanen S, Turunen M, Lehtonen R, Hallikas O, Vanharanta S, Kivioja T, Bjorklund M, Wei G, Yan J, Niittymaki I, et al. 2009. The common colorectal cancer predisposition SNP rs6983267 at chromosome 8q24 confers potential to enhanced Wnt signaling. Nat Genet 41: 885-890.

van de Wetering M, Sancho E, Verweij C, de Lau W, Oving I, Hurlstone A, van der Horn K, Batlle E, Coudreuse D, Haramis AP, et al. 2002. The $\beta$-catenin/TCF- 4 complex imposes a crypt progenitor phenotype on colorectal cancer cells. Cell 111: 241-250.

Wokolorczyk D, Gliniewicz B, Sikorski A, Zlowocka E, Masojc B, Debniak T, Matyjasik J, Mierzejewski M, Medrek $\mathrm{K}$, Oszutowska D, et al. 2008. A range of cancers is associated with the rs6983267 marker on chromosome 8. Cancer Res 68: 9982-9986.

Wood AJ, Severson AF, Meyer BJ. 2010. Condensin and cohesin complexity: The expanding repertoire of functions. Nat Rev Genet 11: 391-404.

Wright JB, Brown SJ, Cole MD. 2010. Upregulation of c-MYC in cis through a large chromatin loop linked to a cancer risk-associated single-nucleotide polymorphism in colorectal cancer cells. Mol Cell Biol 30: 1411-1420.

Yeager M, Orr N, Hayes RB, Jacobs KB, Kraft P, Wacholder S, Minichiello MJ, Fearnhead P, Yu K, Chatterjee N, et al. 2007. Genome-wide association study of prostate cancer identifies a second risk locus at 8q24. Nat Genet 39: 645649.

Yeager M, Xiao N, Hayes RB, Bouffard P, Desany B, Burdett L, Orr N, Matthews C, Qi L, Crenshaw A, et al. 2008. Comprehensive resequence analysis of a $136 \mathrm{~kb}$ region of human chromosome 8q24 associated with prostate and colon cancers. Hum Genet 124: 161-170. 


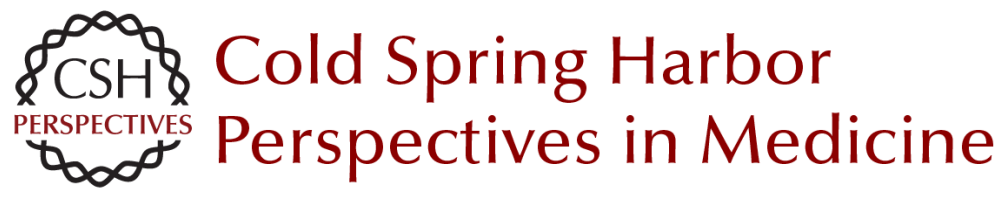

\title{
MYC Association with Cancer Risk and a New Model of MYC-Mediated Repression
}

\author{
Michael D. Cole
}

Cold Spring Harb Perspect Med 2014; doi: 10.1101/cshperspect.a014316

\section{Subject Collection MYC and the Pathway to Cancer}

MYC Cofactors: Molecular Switches Controlling

Diverse Biological Outcomes Stephen R. Hann

MYC Association with Cancer Risk and a New Model of MYC-Mediated Repression Michael D. Cole

MYC and the Art of MicroRNA Maintenance James N. Psathas and Andrei Thomas-Tikhonenko

MYC Activation Is a Hallmark of Cancer Initiation and Maintenance Meital Gabay, Yulin Li and Dean W. Felsher

MYC and Mitochondrial Biogenesis Fionnuala Morrish and David Hockenbery

Synthetic Lethal Screens as a Means to Understand and Treat MYC-Driven Cancers Silvia Cermelli, In Sock Jang, Brady Bernard, et al.

An Overview of MYC and Its Interactome Maralice Conacci-Sorrell, Lisa McFerrin and Robert N. Eisenman

Socializing with MYC: Cell Competition in Development and as a Model for Premalignant Cancer

Laura A. Johnston
MYC and the Control of Apoptosis Steven B. McMahon

Therapeutic Strategies to Inhibit MYC Michael R. McKeown and James E. Bradner

MYC and the Control of DNA Replication David Dominguez-Sola and Jean Gautier

MYC Regulation of Cell Growth through Control of Transcription by RNA Polymerases I and III Kirsteen J. Campbell and Robert J. White

MYC Degradation Amy S. Farrell and Rosalie C. Sears

MYC and Transcription Elongation Peter B. Rahl and Richard A. Young

c-MYC-Induced Genomic Instability Alexandra Kuzyk and Sabine Mai

Oncogenic Mechanisms in Burkitt Lymphoma Roland Schmitz, Michele Ceribelli, Stefania Pittaluga, et al.

For additional articles in this collection, see http://perspectivesinmedicine.cshlp.org/cgi/collection/ 